\title{
Equilibrio trabajo-familia: corresponsabilidad familiar y autoeficacia parental en trabajadores de una empresa chilena*
}

\author{
Work-Family Balance: Working Family and Parental \\ Self-Efficacy in Chilean Company Workers
}

Alejandra Ortega Farías

Dayana Rodríguez Soto

Universidad de Talca, Chile

Andrés Jiménez Figueroa** Universidad de Santiago, Universidad de Talca, Chile

Recibido: 19 de febrero de 2012

Revisado: 22 de mayo de 2012

Aceptado: 18 de agosto de 2012

\section{Resumen}

En el artículo se analizan las diferencias entre equilibrio trabajo-familia, corresponsabilidad y autoeficacia parental en trabajadores de una empresa chilena. A 224 trabajadores les fueron aplicados tres instrumentos: el cuestionario de interacción trabajofamilia, el cuestionario de participación en el trabajo familiar y la escala de autoeficacia parental. Se observa que no existe diferencia en el nivel de equilibrio trabajo-familia entre hombres y mujeres ( $t$ $[222]=-0,83, p>0,05)$. En corresponsabilidad se evidencian diferencias estadísticamente significativas $(\mathrm{t}$ $[220,28]=-7,27, p<0,05)$, indicando que las mujeres realizan más tareas en el hogar, a pesar de su jornada laboral. En relación con la autoeficacia parental existen diferencias entre hombres y mujeres ( $t$ [215] $=-8,76, p<0,05$ ), indicando que las mujeres se perciben a sí mismas como mejores madres.

Palabras clave: equilibrio trabajo-familia, corresponsabilidad familiar, autoeficacia parental, funcionamiento familiar.

Artículo de investigación. Este trabajo forma parte del programa de investigación “Calidad de Vida y Ambientes Saludables” (2007) de la Universidad de Talca, Facultad de Psicología.

** Correspondencia: Andrés Jiménez Figueroa, psicólogo, magíster en Administración y Dirección de Recursos Humanos, Universidad de Santiago, Chile, Facultad de Psicología de la Universidad de Talca. Correo electrónico: anjimenez@utalca.cl 


\section{Abstract}

This article analyzes the differences between work-family balance, joint responsability and parental self-efficacy in employees of a Chilean company. 224 professional workers were administered three instruments: the work-family interaction questionnaire, the participation in family work questionnaire and the parental self-efficacy scale. It is observed that there is no difference in the level of work-family balance between men and women ( $t 222]=-0.83, p>0,05)$. On joint responsibility, there are evident statistically differences (t $[220,28]=-7,27, p<0,05)$, indicating that women do more housework, despite their working day. Regarding parental self-efficacy, there are differences between men and women ( $t[215]=-8,76 p<0,05)$, indicating that women perceive themselves as better mothers.

Keywords: work-family balance, family joint responsibility, parental self-efficacy, family functioning.

\section{Introducción}

El trabajo y la familia son dos esferas fundamentales en el desarrollo del ser humano, caracterizadas por ser interdependientes, pues una influye sobre la otra (Debeljuh y Jáuregui, 2004; Jiménez y Moyano, 2008). A lo largo de muchos años, el rol que ha ejercido la mujer dentro de la sociedad se ha relacionado con labores domésticas y de crianza de los hijos, mientras que el del hombre tiene directa relación con el de proveedor y sostenedor de la familia. Sin embargo, en las últimas décadas esta concepción se ha ido transformando de manera paulatina, debido a los cambios en la estructura y dinámica familiar, el aumento de hogares monoparentales, el acceso a una mayor educación por parte de la mujer y la flexibilización en las condiciones de trabajo (Cifre y Salanova, 2004; Román, Avendaño y Tomicic, 2004; Jiménez y Moyano, 2008). Estos cambios han generado que dentro de las familias ocurran una serie de transformaciones, provocando a su vez la redefinición de las pautas de provisión económica en los hogares y la desnaturalización de cierta estructuración de las relaciones sociales de género (Faur, 2006).

Asimismo, la incorporación de la mujer al mercado laboral generó que dentro de la conformación familiar ocurrieran cambios, los cuales tienen directa relación con el equilibrio que ambos padres deben tener respecto a sus obligaciones laborales y familiares. En Chile, el concepto de familia ha ido cambiando paulatinamente, entre otras cosas, debido al acceso de la mujer a un trabajo remunerado fuera del hogar y al aumento de los matrimonios con contrayentes que tienen edades más tardías y más años de educación formal. Ambos fenómenos han ocasionado que los niños ingresen precozmente a algún tipo de cuidado institucional o permanezcan con sus otros parientes. Con ello se contribuye a que la familia comparta sus roles clásicos y la función educativa de los menores (Vargas, 2001).

En este sentido, Kirchmeyer (2000) considera el equilibrio como la satisfacción en todos los dominios de vida, lo que requiere de recursos personales, energía, tiempo y compromiso para cumplir bien en todos los dominios, ya sea en el trabajo o en la vida familiar. Lo que se pretende, entonces, es que ambos padres trabajen en conjunto para la consecución de una meta en común: la crianza de sus hijos. Es en este punto donde entra el término "corresponsabilidad parental", que según Paterna y Martínez (2009) se logra definir por medio de tres componentes principales: compromiso, es decir, tiempo en interacción cara a cara con el niño; accesibilidad: estar física y psicológicamente presente para atender las necesidades del niño; y responsabilidad: asumir las tareas de cuidado y bienestar día a día.

En relación con lo anterior se puede exponer que la percepción de hombres y mujeres respecto a la corresponsabilidad parental ha ido cambiando a 
lo largo de las generaciones. Es habitual que hoy las mujeres incorporen a sus maridos en labores referentes a la crianza de sus hijos; sin embargo, aún está en el imaginario colectivo que las mujeres participan más de estos procesos. La autoeficacia parental se refiere a las creencias personales sobre las propias capacidades para lograr ser un "buen padre". En este sentido se plantea que los padres que creen en sus propias habilidades se sentirán generalmente más satisfechos y capaces de hacer lo necesario para perseverar y lograr una tarea determinada; ello incluye tener expectativas reales y ser capaces de percibirse a sí mismos como padres competentes (Kendall y Bloomfield, 2005; Farkas-Klein, 2008).

Con base en lo señalado, se hace relevante destacar la importancia que tienen los dominios del trabajo y la familia en la vida de las personas, pues cuando se es padre, con todas las exigencias y responsabilidades que ello implica, la tarea de equilibrar estos dos dominios se complejiza aún más. Algunos estudios muestran que la angustia emocional que produce la dificultad de compatibilizar las demandas y responsabilidades del trabajo y la familia se relacionan con problemas entre padres e hijos y con conductas negativas en estos (Lau, 2009; Valk y Srinivasan, 2011).

Por su parte, existe evidencia que señala que hombres y mujeres no compatibilizan las demandas de ambos dominios de la misma forma, en cuanto al tiempo, nivel de compromiso y equilibrio en la satisfacción. Estos componentes contribuyen a lograr un ajuste y equilibrio entre el trabajo y la familia; es decir, los hombres no dedicarían la misma cantidad de horas que las mujeres al trabajo doméstico, tampoco existiría el mismo nivel de compromiso psicológico y tendrían distintos niveles de satisfacción en el trabajo y el hogar, presentando un menor nivel de ajuste en ambos mundos (Greenhaus, Collins y Shaw, 2003).

Pese a lo anterior, Aguirre y Martínez (2006) afirman que no existe diferencia en los niveles de conflicto experimentados por ambos sexos, argumentando que esto se debe a un cambio en el modelo de pareja, es decir, la evolución del modelo tradicional a un modelo más igualitario, en el que ambos desarrollan una carrera profesional y comparten sus responsabilidades familiares. Ahora bien, independientemente del género, ciertos estudios argumentan que las personas que experimentan estrés en el trabajo tienen interacciones hostiles dentro del núcleo familiar, respondiendo con más sanciones a los comportamientos de sus hijos. Además, la investigación sugiere que aquellos que están físicamente y emocionalmente fatigados por el trabajo tienden a ser menos sensibles, participativos y solidarios con sus hijos. Esto tiene directa relación con el término "autoeficacia parental", entendida como un factor importante en la elección de las conductas que los padres utilizan en la crianza de sus hijos, los esfuerzos que realizan para adaptarse a las diversas situaciones que se presentan y la perseverancia que muestran ante los obstáculos (Lim y Leng, 2003; Jones y Prinz, 2005).

En este sentido, el conflicto que se puede dar al tratar de conciliar el trabajo y familia presenta una correlación significativa y negativa con la calidad percibida en la interacción con los hijos y con la autoeficacia parental; es decir, los niveles más altos de conflicto trabajo-familia están asociados a percepciones más bajas en la calidad de la interacción con los hijos y en la autoeficacia de los padres. Por tanto, al tener altos niveles de conflicto entre ambos dominios, se vería afectada la calidad de la interacción entre padres e hijos y el sentimiento de autoeficacia que los primeros experimentan (Gali, Weisel y Tzuk, 2007; Lau, 2009).

En síntesis, las experiencias vividas en un dominio tenderían a afectar al otro y, en consecuencia, la autoeficacia de los padres y la interacción que estos tienen con sus hijos. En este contexto hay que señalar que si los padres creen en sus propias habilidades se sentirán más satisfechos y capaces de hacer lo necesario para perseverar y lograr una tarea determinada, ello traería consigo tener expectativas reales $y$, además, ser capaces de percibirse a sí mismos como padres competentes (Farkas-Klein, 2008).

Respecto a este tema, las esposas suelen informar emociones negativas hacia el trabajo doméstico, 
incluyendo estrés, frustración, cansancio y aburrimiento (Kawamura y Brow, 2010). Sin embargo, estos sentimientos negativos hacia el trabajo del hogar no se traducen en percepciones de injusticia en la división de las tareas, lo cual revela una discrepancia importante entre el comportamiento individual y la dinámica emocional (Kawamura y Brow, 2010). En el caso de las mujeres casadas y con independencia económica, se señala que son ellas las que realizan la mayor parte de la mano de obra familiar, gastando un promedio de 19 horas por semana en las tareas domésticas, en comparación con los hombres, que solo dedican 10 horas a la semana (Kawamura y Brow, 2010).

La presente investigación pretende determinar las diferencias en cuanto a los niveles de equilibrio trabajo-familia, corresponsabilidad familiar y autoeficacia parental, considerando las posibles diferencias entre hombres y mujeres, lo que puede servir de base para ayudar a fomentar las políticas de conciliación trabajo-familia desde una perspectiva de igualdad de género

\section{Método}

\section{Muestra}

El universo de trabajadores de la empresa corresponde a 310 trabajadores, de los cuales el 55\% son mujeres y el $45 \%$ son hombres. Su segmento etario tiene la siguiente correspondencia: $25 \%$ son menores de 30 años; 46 tienen entre 31 y 40 años; $20 \%$ tienen entre 41 y 50 años; $9 \%$ tienen edades superiores a los 50 años. Para esta investigación se utilizó finalmente una muestra de 224 trabajadores, de los cuales 93 eran hombres y 131 eran mujeres, cuya característica principal era que tenían hijos.

\section{Instrumentos}

\section{Cuestionario de interacción trabajo- familia (SWING)}

Este cuestionario está compuesto por 22 ítems tipo Likert, distribuidos en 4 subescalas: interacción negativa trabajo-familia, interacción negativa familia-trabajo, interacción positiva trabajo- familia e interacción positiva familia-trabajo. Este cuestionario posee propiedades psicométricas adecuadas, con buena consistencia interna y con valores que se sitúan entre 0,77 y 0,89 (Moreno, Sanz, Rodríguez y Geurts, 2009).

\section{Cuestionario de participación en el trabajo familiar}

Para medir la variable corresponsabilidad familiar se utilizó el cuestionario de participación en el trabajo familiar de los autores Bartau, Etxebarría y Maganto (2003). Este cuestionario está compuesto por 22 tareas del hogar, y sus respuestas varían de 0 a 2 , dependiendo de la frecuencia en la que se realice $(0=$ nunca, $1=$ a veces y $2=$ siempre $)$. Estas están enfocadas a medir los siguientes tipos de labores: realización de "mis cosas", las "tareas por otros", las "tareas accesorias" y las "tareas fundamentales". A partir de estas dimensiones se construye el índice de participación individual, debido a que cada una de las tareas posee una importancia distinta en la corresponsabilidad.

\section{Escala de Evaluación Parental (EEP)}

Es un cuestionario autoadministrado, creado para evaluar la satisfacción y los sentimientos de autoeficacia respecto a la maternidad. Inicialmente, la EEP se compuso de 20 ítems, los que fueron chequeados en una población de mujeres chilenas, a partir de lo cual se obtuvo una adecuada consistencia interna, mientras que el análisis factorial arrojó dos factores que explican el 63\% de la varianza. La versión final de la EEP considera 10 ítems y entrega un puntaje global de la autoeficacia materna, con lo que se obtiene una consistencia interna de 0,85 (Farkas-Klein, 2008).

\section{Procedimiento}

Se realizó contacto con la empresa y se entregaron los instrumentos en los lugares acordados, entre agosto y octubre de 2011. Los instrumentos fueron administrados a 224 trabajadores de ambos sexos conjuntamente, con su respectivo consentimiento en las distintas sucursales. La duración total de la aplicación de los 3 cuestionarios por persona fue de 20 minutos. Finalmente, para el análisis de los datos se utilizaron procedimientos 
estadísticos descriptivos, además de comparación de medias por medio de pruebas $T$ para muestras independientes. Se realizaron también procedimientos estadísticos correlacionales bivariados, mediante el programa estadístico SPSS 15.0.

\section{Resultados}

Respecto a la variable equilibrio trabajo-familia se observa que los hombres presentan una media de 3,05 ( $D E=0,63)$, en tanto las mujeres presentan una media de $3,12(\mathrm{DE}=0,61)$. Por otra parte, considerando los factores de la variable equilibrio trabajo-familia, se logra determinar que los hombres presentan una puntuación media de 3,04 (DE $=0,70$ ), mientras que las mujeres poseen una media de 3,07 $(D E=0,68)$ en la dimensión que indica una interacción negativa trabajo-familia (tabla 1).

Tabla 1.

Análisis descriptivo del equilibrio trabajo-familia por factores por sexo

\begin{tabular}{|c|c|c|c|c|c|c|}
\hline \multirow{9}{*}{ 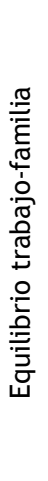 } & Dimensión & Sexo & $\mathbf{N}$ & M & $\begin{array}{c}\text { Desv. } \\
\text { Típ. }\end{array}$ & $\begin{array}{c}\text { Error } \\
\text { Típ. }\end{array}$ \\
\hline & ETFneg TF & $\mathrm{H}$ & 93 & 3,04 & 0,7 & 0,07 \\
\hline & & M & 131 & 3,07 & 0,68 & 0,06 \\
\hline & ETFneg FT & $\mathrm{H}$ & 93 & 3,25 & 0,8 & 0,08 \\
\hline & & M & 131 & 3,33 & 0,75 & 0,07 \\
\hline & ETFpos TF & $\mathrm{H}$ & 93 & 2,87 & 0,64 & 0,07 \\
\hline & & M & 131 & 2,95 & 0,65 & 0,06 \\
\hline & ETFpos FT & $\mathrm{H}$ & 93 & 3,1 & 0,78 & 0,08 \\
\hline & & M & 131 & 3,21 & 0,74 & 0,06 \\
\hline
\end{tabular}

Fuente: autores.

Mediante la prueba T para muestras independientes y asumiendo igualdad de varianza $(F=016$; $p>0,05)$, se puede determinar que no existen diferencias significativas en el nivel de equilibrio trabajo-familia entre hombre y mujeres (t [222] = $-0,83 ; p>0,05)$ (tabla 2).
Tabla 2.

Diferencias de media en la dimensión equilibrio trabajo-familia

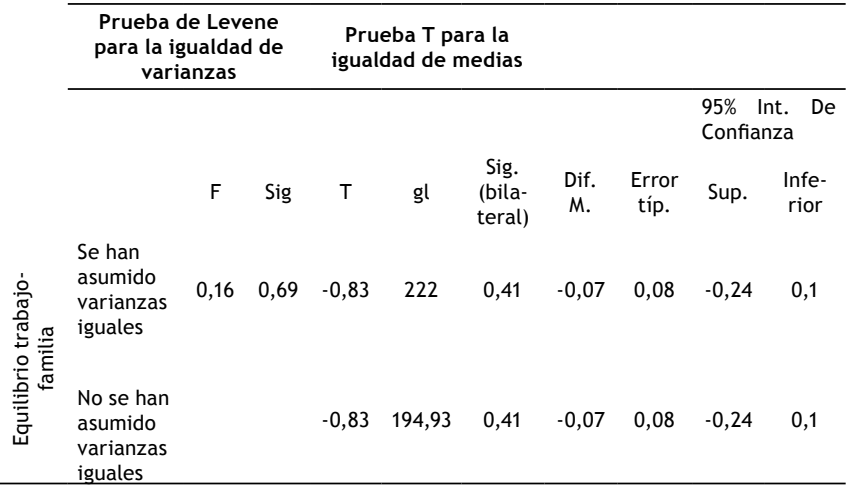

Fuente: autores.

En cuanto a las dimensiones de la variable equilibrio trabajo-familia se logra determinar que tanto en la dimensión de interacción negativa trabajofamilia como en la dimensión de interacción negativa familia-trabajo no existen diferencias estadísticamente significativas entre hombres y mujeres (t [222] $=-0,38 ; p>0,05) ;$ (t [222] $=-0,074 ; p>$ $0,05)$, respectivamente. De igual manera, en las dimensiones de interacción positiva trabajo-familia e interacción positiva familia-trabajo no se encuentran diferencias estadísticamente significativas entre hombres y mujeres (t $[222]=-0,85 ; p$ > $0,05)$; $(\mathrm{t}[222]=-1,10 ; p>0,05)$, respectivamente.

Respecto a los resultados en la variable corresponsabilidad se observa que los hombres presentan una media de 28,23 ( $D E=5,78$,) en tanto las mujeres presentan una media de $34,66(\mathrm{DE}=$ 7,46). En relación con el índice de participación familiar, los hombres presentan una media de 11,02 ( $D E=2,22$ ), y las mujeres poseen una media de 13,38 (DE = 2,73) (tabla 3). 
Tabla 3.

Análisis descriptivo de corresponsabilidad por dimensión y según sexo

\begin{tabular}{|c|c|c|c|c|c|c|}
\hline \multirow{9}{*}{ 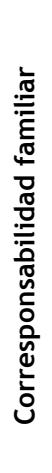 } & Dimensión & Sexo & $\mathrm{N}$ & $M$ & $\begin{array}{l}\text { Desv. } \\
\text { Típ. }\end{array}$ & $\begin{array}{c}\text { Error } \\
\text { Típ. }\end{array}$ \\
\hline & \multirow{2}{*}{$\begin{array}{l}\text { Tareas } \\
\text { propias }\end{array}$} & $\mathrm{H}$ & 93 & 1,37 & 0,37 & 0,04 \\
\hline & & $M$ & 131 & 1,66 & 0,61 & 0,05 \\
\hline & \multirow{2}{*}{$\begin{array}{l}\text { Tareas } \\
\text { por otros }\end{array}$} & $\mathrm{H}$ & 93 & 1,11 & 0,37 & 0,04 \\
\hline & & M & 131 & 1,46 & 0,49 & 0,04 \\
\hline & \multirow{2}{*}{$\begin{array}{l}\text { Tareas } \\
\text { accesorias }\end{array}$} & $\mathrm{H}$ & 93 & 1,21 & 0,34 & 0,04 \\
\hline & & $M$ & 131 & 1,32 & 0,41 & 0,04 \\
\hline & \multirow{2}{*}{$\begin{array}{l}\text { Tareas } \\
\text { fund. }\end{array}$} & $\mathrm{H}$ & 93 & 1,67 & 0,38 & 0,04 \\
\hline & & M & 131 & 2,05 & 0,42 & 0,04 \\
\hline
\end{tabular}

Fuente: autores.

A partir de los datos obtenidos mediante la prueba $T$ para muestras independientes y asumiendo desigualdad de varianza $(F=17,02 ; p<0,05)$, se puede determinar que existen diferencias estadísticamente significativas en la corresponsabilidad desarrollada entre hombre y mujeres (t $[220,28]$ $=-7,27 ; p<0,05)$ (tabla 4).

Tabla 4.

Comparación de medias de corresponsabilidad según el sexo

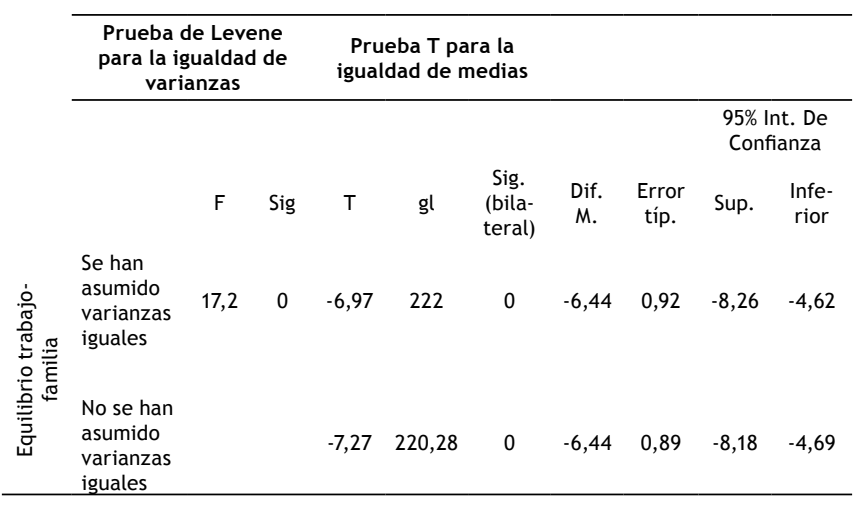

Fuente: autores.

De acuerdo con el índice de participación familiar y a través de la prueba $T$ para muestras independientes, se asume desigualdad de varianza $(F=$ $14,48 ; p<0,05)$ y se determina que existen diferencias estadísticamente significativas en este índice de participación familiar entre hombre y mujeres (t $[217,96]=-7,11 ; p<0,05)$ (tabla 5).

Tabla 5.

Comparación de medias del índice de participación en corresponsabiidad según el sexo

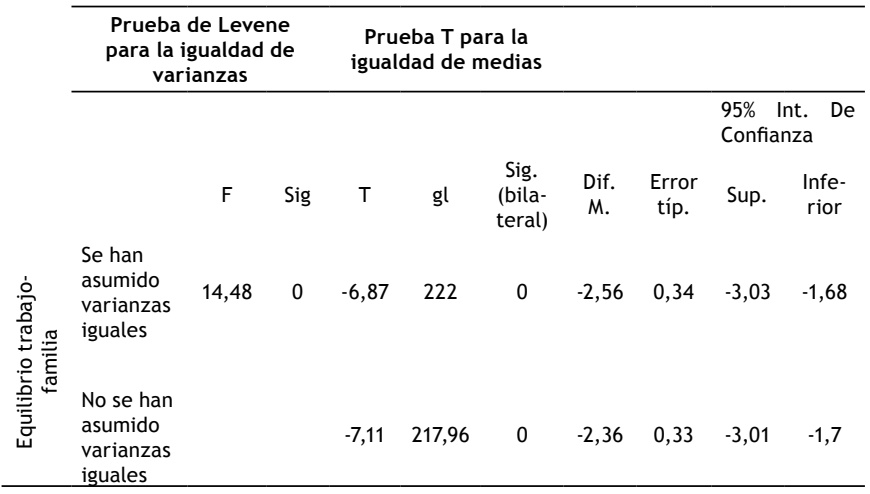

Fuente: autores.

En la variable autoeficacia parental, los resultados señalan que los hombres presentan una media de 6,10 ( $D E=1,14)$, mientras las mujeres presentan una media de $7,77(D E=1,67)$. Por medio de la prueba $T$ para muestras independientes y desigualdad de varianza $(F=16,51 ; p<0,05)$, se puede determinar que existen diferencias estadísticamente significativas en la autoeficacia parental entre hombres y mujeres (t [215] $=-8,76 p$ $<0,05)$ (tabla 6).

Tabla 6

Comparación de medias de autoeficacia parental según el sexo

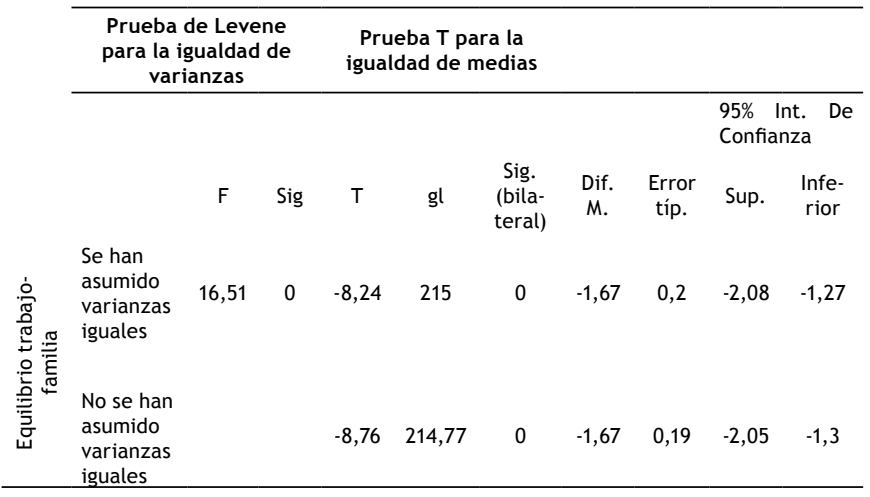

Fuente: autores. 
Respecto a la relación entre las variables equilibrio trabajo-familia y autoeficacia parental, puede señalarse que esta es estadísticamente significativa y de intensidad moderada $(r=0,412 ; p<$ $0,01)$. Lo mismo ocurre entre la variable corresponsabilidad y autoeficacia parental, que presenta una relación estadísticamente significativa de intensidad baja $(r=0,203 ; p<0,01)$. Finalmente, respecto a la variable corresponsabilidad, utilizando su índice de participación, se evidencia una relación estadísticamente significativa con la variable autoeficacia parental $(r=0,188 ; p<0,01)$ (tabla 7).

Tabla 7.

Relación entre equilibo trabajo-familia, autoeficacia parental, corresponsabilidad e índice de participación

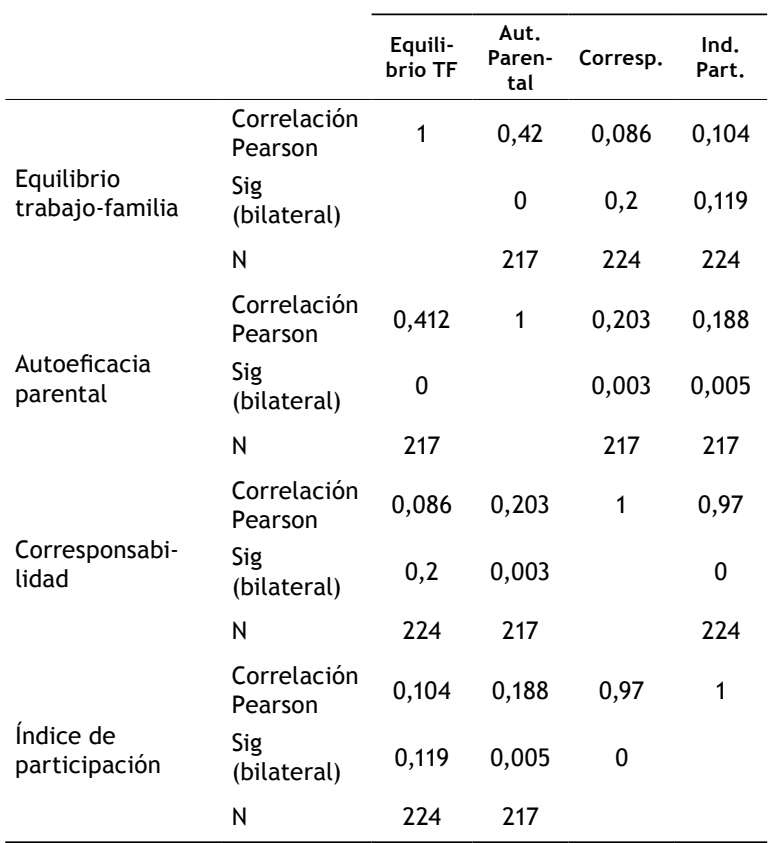

La correlación es significativa al nivel 0,01 (bilateral)

Fuente: autores.

\section{Discusión}

La incorporación de la mujer en el mercado laboral ha generado diversos cambios en el interior de las familias, modificando su estructura y organización; además, las parejas han tenido que compatibilizar sus responsabilidades laborales y familiares, provocando equilibrio o conflicto entre ambas esferas. Esta nueva reestructuración ha repercutido en la distribución de las tareas propias del hogar y en el tiempo que la pareja le dedica a sus hijos (Clark, 2000; Valdés y Olavarría, 1998; Jiménez y Moyano, 2008).

Debeljuh y Jáuregui (2004) señalan que una forma de equilibrar las demandas del trabajo y la familia debe provenir tanto del propio individuo, como del Estado y de la empresa, los cuales deben intervenir de manera simultánea. Por tanto, plantean que los trabajadores tendrían que idear sus propias estrategias para hacer compatibles las demandas tanto del ámbito del trabajo como del familiar.

En esta investigación se encontraron diferencias significativas en la corresponsabilidad parental que perciben hombres y mujeres trabajadores. Referente a esto, el Instituto Nacional de Estadísticas (2008) devela la inequidad de género existente en la distribución de roles en el interior de los hogares, aun cuando la mujer se ha incorporado al sistema laboral y pasa gran parte del tiempo fuera de su casa, realizando una doble jornada. Por tanto, sigue siendo la mujer la responsable de la mayor parte del trabajo doméstico, gastando un promedio de 19 horas por semana en las tareas del hogar, en comparación con los hombres, que solo dedican 10 horas a la semana (Artázcoz et ál., 2001; Kawamura y Brow, 2010). Además, de acuerdo con el índice de participación arrojado en este estudio, se observan diferencias significativas entre ambos sexos, indicando que son las mujeres quienes realizan más tareas domésticas, tales como hacer las camas, limpiar el baño después de ducharse, recoger las cosas que otro ha utilizado, sacar la basura, cocinar, planchar, limpiar la casa, hacer las compras, entre otras.

Se constatan diferencias significativas en la autoeficacia parental que perciben hombres y mujeres trabajadores. Esto se puede explicar si se toma el contexto en cual están inmersas todas las personas, debido a que culturalmente, dentro de una familia, quien ocupa el rol de estar a cargo del cuidado de los hijos es la madre, mientras que 
el padre posee el rol de proveedor. A partir de esto, diversos autores concuerdan en que transcurridos los primeros años de vida del bebé, las funciones de los padres difieren entre sí, conllevando que el rol del cuidado del bebé posea connotaciones diferentes en padres y madres. Esto ha generado que el estudio de la eficacia personal considere dominios distintos en ambos, provocando que las madres, al pasar más tiempo con sus hijos, se evalúen de una forma más positiva (Farkas-Klein, 2008).

Aun cuando ambos padres participan en el trabajo remunerado, tanto por elección como por necesidad, la familia como sistema social debe invertir tiempo y energía en actividades tanto en el trabajo remunerado como en el trabajo familiar, correspondiente al cuidado de los niños y a las tareas del hogar (Bartau et ál., 2003); es decir, la conciliación trabajo-familia implica coordinar las demandas tanto del ámbito público como privado, con un mínimo conflicto de roles. En el ámbito privado toma importancia la corresponsabilidad, que hace referencia al tiempo que invierte la pareja en el cuidado de los hijos y en los quehaceres del hogar, en donde ambos deben involucrarse activamente.

De acuerdo con esto, se planteó la existencia de una correlación entre las variables equilibrio trabajo-familia y corresponsabilidad parental, pues ambas abordan el mundo privado de las personas, pero en distintos niveles. Sin embargo, en este estudio no se evidencia tal relación, lo que se puede deber a que culturalmente la mujer siempre ha estado relacionada con el trabajo doméstico; y si bien la participación del hombre en el hogar ha aumentado, lo hace solo apoyando la labor de esta, tomando un papel secundario. Esto se respalda con la afirmación de Rodríguez y Larrañaga (2011), que plantean que la carga de las tareas que se llevan a cabo dentro del hogar continúa siendo primordialmente del sexo femenino; situación que se da incluso en sociedades en las que el hombre tiene una alta participación en el trabajo doméstico.

Finalmente, hay que mencionar que la distribución desigual del trabajo dentro de la casa es a menudo justificada por las expectativas sociales, pues se pone a los hombres como sostén de la familia y a las mujeres como amas de casa. En este sentido, el menor trabajo que ejerce el marido en las labores del hogar se justifica por la demanda de su rol de proveedor, es decir, trabaja fuera del hogar y contribuye con los ingresos de este. Además, ciertas investigaciones sugieren que los hombres, en comparación con las mujeres, se muestran poco dispuestos a organizar su tiempo libre para colaborar con los quehaceres de sus parejas (Cifre y Salanova, 2004; Kawamura y Brow, 2010).

La relación entre el trabajo y la familia tiene dos visiones: una negativa, en la cual se presenta un conflicto entre ambas esferas; y una positiva, en la que lo primordial es el equilibrio entre estas. En relación con la primera mirada, estudios argumentan que independiente del género, las personas que experimentan estrés en el trabajo tienen interacciones hostiles dentro del núcleo familiar, respondiendo con más sanciones a los comportamientos de sus hijos. Además, la investigación sugiere que aquellos que están física y emocionalmente fatigados por el trabajo tienden a ser menos sensibles, participativos y solidarios con sus hijos. Esto tiene directa relación con el término "autoeficacia parental", entendida como un factor importante en la elección de las conductas que los padres utilizan en la crianza de sus hijos, los esfuerzos que realizan para adaptarse a las diversas situaciones que se presentan y la perseverancia que muestran ante los obstáculos (Lim y Leng, 2003).

Lo anterior da cuenta de cómo ambas esferas se complementan e influencian, es decir, el bienestar en un ámbito llevará a presentar conductas más positivas en la otra esfera; por ejemplo, si la persona ha logrado conciliar su vida laboral con la familiar, estará más dispuesta y atenta a los requerimientos de sus hijos. Estas conductas hacen que los padres se vayan evaluando a sí mismos en su rol. Es aquí en donde entra el tema de la autoeficacia, ya que si los padres creen en sus propias habilidades, se sentirán más satisfechos y capaces de hacer lo necesario para perseverar y lograr una tarea determinada, lo que traería consigo 
tener expectativas reales y, además, ser capaces de percibirse a sí mismos como padres competentes (Farkas-Klein, 2008).

La investigación presenta algunas limitaciones, las cuales pueden ser abordadas en futuros estudios. En primer lugar, se considera relevante, para futuras investigaciones, contrastar estos resultados con una empresa que no promueva este tipo de políticas, con el objetivo de saber si realmente son estas las que conllevan un equilibro entre ambas esferas y actúan en las otras variables o, por el contrario, simplemente son producto de factores externos ajenos a la empresa. Asimismo, sería aconsejable, para complementar cualitativamente la capacidad de análisis ante los fenómenos evaluados, que se incluyan las apreciaciones y los argumentos que dan las personas ante los temas estudiados.

Finalmente, por medio de este estudio se entregan aportes relevantes a las temáticas abordadas; uno de ellos es que se estudió el ámbito privado de los trabajadores, involucrándose en la dinámica familiar y en la distribución de las tareas que se dan en el interior del hogar. Esto es debido a que tanto a nivel gubernamental como a nivel de empresa solo se han implementado una serie políticas tendentes a conciliar la vida laboral y familiar, pero no han logrado involucrarse en el mundo privado de sus trabajadores, conociendo y llevando a cabo acciones que promuevan la corresponsabilidad y la autoeficacia parental.

\section{Referencias}

Aguirre, Z. y Martínez, M. P. (2006). Influencia de la situación laboral en el ajuste familia-trabajo. Mapfre Medicina, 17(1), 14-24.

Artázcoz, L., Borrell, C., Rohlfs, I., Beni, C., Moncada, A. y Benach, J. (2001). Trabajo doméstico, género y salud en población ocupada. Gac Sanit, 15(2), 150-153.

Bartau, I., Etxebarria, J. y Maganto, J. (2003). La participación en el trabajo familiar: un reto educativo y social. Revista Electrónica de Investigación y Evaluación Educativa, 9(2), 160-183.

Cifre, E. y Salanova, M. (2004). Estrategias de conciliación familia/trabajo: buscando la calidad de vida. Estudios Financieros, 259, 11-154.

Clark, S. (2000). Work cultures and work/family balance. Journal of Vocational Behavior, 58, 348- 365.

Debeljuh, P. y Jáuregui, K. (2004). Trabajo y familia: hacia una cultura familiar amigable en el contexto latinoamericano. Esan, Cuadernos de Difusión, 16, 91-102.

Farkas-Klein, C. (2008). Escala de Evaluación Parental (EEP): desarrollo, propiedades psicométricas y aplicaciones. Universitas Psychologica, 7(2), 457-467.

Faur, E. (2006). Género, masculinidades y políticas de conciliación familia-trabajo. Nómadas. Recuperado de http://redalyc.uaemex.mx/ src/inicio/ArtPdfRed.jsp?iCve $=105116598012$

Gali, R., Weisel, A. \& Tzuk, K. (2007). Work-family conflict within the family: crossover effects, perceived parent child interaction quality, parental selfefficacy, and life role attributions. Journal of Career Development, 34, 79-100.

Greenhaus, J., Collins, K. \& Shaw, J. (2003). The relation between work-family balance and quality of life. Journal of Vocational Behavior, 63, 510- 531.

Instituto Nacional de Estadísticas (2008). Encuesta experimental sobre uso del tiempo en el gran Santiago. Recuperado de http://www.ine.cl/ canales/sala_prensa/noticias/2008/mayo/ pdf/presentacion300508.pdf

Jiménez, A. y Moyano, E. (2008). Factores laborales entre trabajo y familia: medios para mejorar la calidad de vida. Revista Universum, 1(23), 116-133. 
Jones, T. L. \& Prinz, R. J. (2005). Potential roles of parental self-efficacy in parent and child adjustment: a review. Clinical Psychology Review, 25, 341-363.

Kawamura, S. \& Brown, S. (2010). Mattering and wives' perceived fairness of the division of household labor. Social Science Research, 39, 976-986.

Kendall, S. \& Bloomfield, L. (2005). Developing and validating a tool to measure parenting self-efficacy. Journal of Advanced Nursing, 51(2), 174-181.

Kirchmeyer, C. (2000). Work-life initiatives: greed or benevolence regarding workers' time? En C. L. Cooper \& D. M. Rousseau (Eds.). Trends in Oganizational Behaviour (pp. 79-93). Chichester, UK: Wiley.

Lau, Y. (2009). The impact of fathers' work and family conflicts on children's self- esteem: the Hong Kong case. Social Indicators Research, 95, 363-376.

Lim, V. y Leng, G. (2003). Effects of parental job insecurity and parenting behaviors on youths self-efficacy and work attitudes. Journal of Vocational Behavior, 63(1), 86-98.

Moreno, B., Sanz, A., Rodríguez, A. y Geurts, S. (2009). Propiedades psicométricas de la versión española del Cuestionario de Interacción Trabajo-Familia (SWING). Psicothema, 21, 331-337.
Paterna, C. y Martínez, M. (2009). Influencia de las variables de género en la distribución de las tareas domésticas y de cuidado. Interamerican Journal of Psychology, 43(2), 241-249.

Rodríguez, A. y Larrañaga, M. (s.f.). El tiempo de trabajo y su distribución por sexos en la comunidad autónoma Vasca. Recuperado de http://www.consorcio.org.mx/documentos/ tiempo-trabajo.pdf

Román, J., Avendaño, C. y Tomicic, A. (2004). Conflicto y equilibrio en la relación trabajofamilia: un estudio con mujeres trabajadoras por cuenta propia. Persona y Sociedad, 18(1), 51-76.

Valdés, T. y Olavarría, J. (1998). Masculinidades y equidad de género en América Latina. Santiago de Chile: Flacso-Chile. Recuperado de http://cronopio.flacso.cl/fondo/pub/publi cos/1998/libro/001713.pdf

Valk, R. \& Srinivasan, V. (2011). Workefamily balance of Indian women software professionals: a qualitative study. IIMB Management Review, 23(1), 39-50.

Vargas, N. (2001). Cambios en la familia: repercusiones en la práctica pediátrica. Rev. Chil. Pediatr., 72(2), 77-80. 